This is the post peer-review accepted manuscript of:

Arias-de-Reyna, E., Dardari, D., Closas, P., \& Djuric, P. M. (2018). Estimation Of Spatial Fields Of Nlos/Los Conditions For Improved Localization In Indoor Environments. In 2018 IEEE Statistical Signal Processing Workshop (SSP) (pp. 658-662). https://doi.org/10.1109/SSP.2018.8450840

The published version is available online at: https://doi.org/10.1109/SSP.2018.8450840

C 2018 IEEE. Personal use of this material is permitted. Permission from IEEE must be obtained for all other uses, in any current or future media, including reprinting/republishing this material for advertising or promotional purposes, creating new collective works, for resale or redistribution to servers or lists, or reuse of any copyrighted component of this work in other works 


\title{
ESTIMATION OF SPATIAL FIELDS OF NLOS/LOS CONDITIONS FOR IMPROVED LOCALIZATION IN INDOOR ENVIRONMENTS
}

\author{
Eva Arias-de-Reyna*, Davide Dardari**, Pau Closas ${ }^{\dagger}$, and Petar M. Djuric $c^{\ddagger}$ \\ * Department of Signal Theory and Communications, Universidad de Sevilla, Sevilla (Spain) \\ ** Department of Electrical, Electronic and Information Eng. (DEI) and CNIT, University of Bologna, Cesena, Italy \\ $\dagger$ Department of Electrical and Computer Engineering, Northeastern University, Boston, MA (USA) \\ $\ddagger$ Department of Electrical and Computer Engineering, Stony Brook University, Stony Brook, NY (USA) \\ Email: earias@us.es,davide.dardari@unibo.it, closas@northeastern.edu,petar.djuric@stonybrook.edu
}

\begin{abstract}
A major challenge in indoor localization is the presence or absence of line-of-sight (LOS). The absence of LOS, denoted as non-line-of-sight (NLOS), directly affects the accuracy of any localization algorithm because of the induced bias in ranging. The estimation of the spatial distribution of NLOS-induced ranging bias in indoor environments remains a major challenge. In this paper, we propose a novel crowd-based Bayesian learning approach to the estimation of bias fields caused by LOS/NLOS conditions. The proposed method is based on the concept of Gaussian processes and exploits numerous measurements. The performance of the method is demonstrated with extensive experiments.
\end{abstract}

Index Terms - Indoor localization, crowd sourcing, Gaussian processes, spatial field, NLOS

\section{INTRODUCTION}

Indoor localization systems are a key technology for enabling Location-based Services (LBS) [1]. There are several methodologies for localization including fingerprinting and modelbased methods [2], [3]. Here, we are interested in the latter, where localization algorithms are designed so that a user computes its location in real-time based on its own past and current measurements. In this context, there is the need to specify a model (usually probabilistic) that maps the measurements to geometric quantities (distance, angle, position, etc.) with some uncertainty. The adopted model often depends critically on some (potentially unknown) conditions and/or parameters, which are typically location-dependent such as the LOS/NLOS condition [4][6], or the ranging bias and variance [4], [5], [7]. When a subset of model parameters is unknown, a number of approaches for their improved estimation have been investigated, including methods based on cooperation and learning [8]-[17].

The aforementioned learning schemes typically require a huge amount of measurements to be reliable, which in turn must come from expensive training sessions that become out-of-date after a short time. Alternatively, due to the widespread use of mass market devices with sensing capabilities, mobile crowd

EA has been supported by the Spanish Ministry of Economy and Competitiveness and the Spanish National Research Agency under grant TEC2016-78434-C3-2-R (AEI/FEDER, EU), by the European Union (FEDER) and by Junta de Andalucía (TIC-155). DD has been supported by the European H2020 project XCycle (Grant 635975). PMD has been supported by NSF under Awards CCF-1618999 and CNS-1642965. sensing has emerged as an appealing paradigm to enable largescale applications [18]. In many practical scenarios, one may have measurements acquired by hundreds or thousands of people navigating through an indoor environment in different hours, days and months and which can be exploited for improved localization [19], [20]. In this context one challenging aspect is the "Big data" issue. Considering that in crowd sensing scenarios the number $N$ of measurements grows exponentially, we investigate learning approaches whose memory and computational burden do not increase with $N$.

The main contribution of this article is a novel methodology for range-based indoor localization which relies on estimating a spatial field related to the LOS/NLOS conditions. In particular, in time-based ranging, the NLOS condition gives rise to an estimated range larger than the true distance. Of interest in this paper is the estimation of the spatial field of the bias. Specifically, we propose a method for joint positioning and learning the bias field by exploiting the power of the crowd and whose memory and computational burden do not increase with the number of measurements. The method is based on modeling the bias field by a Gaussian process (GP), where we represent the process in a form that allows for bounded use of memory. GPs have already been used in the literature of cognitive radio for detecting the presence of primary users, e.g., [21]. Our method follows a line of reasoning presented in our previous paper [22], where we have adopted a "loose coupling" approach with two separate estimators, one for the position and one for the field. These estimators "ping-pong" their estimates as new measurements are being acquired. Here we propose a different and improved version of this approach.

While a user moves in the area of interest, the central unit estimates the location and velocity of the user by taking advantage of the estimated field obtained from measurements of previous users. Further, the central unit updates the estimate of the field by the measurements of this user. Thereby, subsequent users can also benefit by using the field for their own localization. We point out that our scheme is capable of accounting for spatial correlation.

The paper is organized as follows. First, in Section II, we provide the problem statement and the adopted model for the spatial fields. In Section III, we present the details of the proposed estimation method. Numerical results that demonstrate the performance of the method are shown in Section IV. Section V contains final conclusions. 


\section{CROWD ENHANCED LOCALIZATION}

\section{II-A. Problem Statement}

Mobile users enter an indoor environment at random times, roam in it along random trajectories, and then leave it. This environment could be, for example, a shopping mall or a business center. The movement of the $k$ th mobile user is modeled by

$$
\mathbf{x}_{t, k}=\mathbf{A}_{k} \mathbf{x}_{t-1, k}+\mathbf{u}_{t, k}
$$

where $\mathbf{x}_{t, k}=\left[\begin{array}{llll}x_{t, k, 1} & x_{t, k, 2} & \dot{x}_{t, k, 1} & \dot{x}_{t, k, 2}\end{array}\right]^{\top}$ is a state vector of the $k$ th user whose elements are the Cartesian coordinates of its location $\left(x_{t, k, 1}\right.$ and $\left.x_{t, k, 2}\right)$ and its velocity components $\left(\dot{x}_{t, k, 1}\right.$ and $\left.\dot{x}_{t, k, 2}\right)$ at discrete time $t ; \mathbf{A}_{k}$ is a $4 \times 4$ known matrix, which models the user's movement; and $\mathbf{u}_{t, k}$, is a random perturbation with zero mean Gaussian distribution, i.e., $\mathbf{u}_{t, k} \sim \mathcal{N}\left(\mathbf{0}, \mathbf{R}_{k}\right)$, with $\mathbf{R}_{k}=\rho_{k}^{2} \mathbf{R}_{0}$, where $\rho_{k}^{2}$ is unknown and user dependent and the $4 \times 4$ matrix $\mathbf{R}_{0}$ is known. The samples $\mathbf{u}_{t, k}$ for different $t$ and $k$ are assumed independent.

While the users move in the indoor area, they acquire measurements of signals (e.g., their time-of-arrival) transmitted by fixed nodes, referred to as anchors, deployed in the environment at known positions $\mathbf{l}^{[n]}, n=1,2, \cdots, N_{a}$. The corresponding distance measurements acquired by the $k$ th user from the $n$th anchor at time $t$ are modeled as

$$
y_{t, k}^{[n]}=\left|\mathbf{P} \mathbf{x}_{t, k}-\mathbf{l}^{[n]}\right|+f^{[n]}\left(\mathbf{x}_{t, k}\right)+\nu_{t, k}^{[n]},
$$

where $n=1,2, \cdots, N_{a}$ and $\left|\mathbf{P} \mathbf{x}_{t, k}-\mathbf{l}^{[n]}\right|$ is the distance between user $k$ and anchor $n$ at time $t$ with $\mathbf{P}$ being a $2 \times 4$ matrix instrumentally used to extract the position components from the full state vector.

The symbol $f^{[n]}\left(\mathbf{x}_{t, k}\right)$ represents a bias field that models position-dependent deviations of the observations from anchor $n$ to the location of the user caused by NLOS / LOS conditions, and $\nu_{t, k}^{[n]}$ is the measurement noise modeled as a zero mean Gaussian random variable $(\mathrm{RV})$ with unknown standard deviation $\sigma_{\nu}^{[n]}$. Recall that there are $N_{a}$ anchors located at $\mathbf{l}^{[n]}, n=1,2, \cdots, N_{a}$, and therefore there are $N_{a}$ bias fields. We denote them by $\mathbf{f}(\mathbf{x})=$ $\left\{f^{[1]}(\mathbf{x}), f^{[2]}(\mathbf{x}), \ldots, f^{\left[N_{\mathrm{A}}\right]}(\mathbf{x})\right\}$.

Next, we assume that the user acquires the measurements from all the anchors at the same time slot, and we denote them by $\mathbf{y}_{t, k}$, where $\mathbf{y}_{t, k}=\left[y_{t, k}^{[1]} y_{t, k}^{[2]} \cdots y_{t, k}^{\left[N_{a}\right]}\right]^{\top}$. When the users enter the environment, based on the received measurements $\mathbf{y}_{t, k}$, the system tracks the users. Much improved tracking can be accomplished if the system knows the $N_{a}$ bias fields.

In this paper, we address the problem of joint tracking the user's position and learning the fields $f^{[n]}(\mathbf{x}), \forall n$, from measurements of the users.

\section{II-B. Model for Spatial Fields}

In absence of specific and accurate models for $f^{[n]}(\mathbf{x})$, we assume that $f^{[n]}(\mathbf{x})$ is a sample from a GP

$$
f^{[n]}(\mathbf{x}) \sim \mathcal{G P}\left(\mu^{[n]}(\mathbf{x}), \kappa^{[n]}\left(\mathbf{x}, \mathbf{x}^{\prime}\right)\right)
$$

with mean $\mu^{[n]}(\mathbf{x})$ and covariance function $\kappa^{[n]}\left(\mathbf{x}, \mathbf{x}^{\prime}\right)$.

Given a set of 2D orthogonal basis functions in the area of interest $\boldsymbol{\psi}(\mathbf{x})=\left[\psi_{1}(\mathbf{x}) \psi_{2}(\mathbf{x}), \ldots, \psi_{J}(\mathbf{x})\right]^{\top}$, we propose to model $f^{[n]}(\mathbf{x})$ by

$$
f^{[n]}(\mathbf{x})=\boldsymbol{\psi}^{\top}(\mathbf{x}) \mathbf{c}^{[n]},
$$

where the vector $\boldsymbol{\psi}(\mathbf{x})$ is of size $J \times 1$, and $\mathbf{c}^{[n]}$ is a $J \times 1$ Gaussian vector of coefficients that corresponds to the $n$th anchor and that is used for modeling the bias field of that anchor. Thus, if $\mathbf{c}^{[n]} \sim \mathcal{N}\left(\boldsymbol{\mu}_{c}^{[n]}, \boldsymbol{\Sigma}_{c}^{[n]}\right)$, then

$$
f^{[n]}(\mathbf{x}) \sim \mathcal{N}\left(\boldsymbol{\psi}^{\top}(\mathbf{x}) \boldsymbol{\mu}_{c}^{[n]}, \boldsymbol{\psi}^{\top}(\mathbf{x}) \boldsymbol{\Sigma}_{c}^{[n]} \boldsymbol{\psi}(\mathbf{x})\right) .
$$

We point out that $\boldsymbol{\mu}_{c}^{[n]}$ and $\boldsymbol{\Sigma}_{c}^{[n]}$ are moments estimated by crowd sensing. We will elaborate more on the process of estimating these moments in the next section.

Thanks to (4), the problem of representing and estimating the bias field $f^{[n]}(\mathbf{x})$ translates into characterizing the vector of coefficients $\mathbf{c}^{[n]}$ that do not depend on $\mathbf{x}$ and that do not increase in size with the increase of the number of measurements. The key to the crowd sensing idea is to use the information collected by all the past users for estimating the means and covariance matrices $\boldsymbol{\mu}_{c}^{[n]}$ and $\boldsymbol{\Sigma}_{c}^{[n]}, \forall n$. In this modeling, we are assuming that the anchor's fields are independent among each of them.

We rewrite (2) by using (4) in a vector-matrix form as follows:

$$
\mathbf{y}_{k}^{[n]}=\mathbf{s}_{k}^{[n]}+\boldsymbol{\Psi}_{T_{k}, k} \mathbf{c}^{[n]}+\boldsymbol{\nu}_{k}^{[n]}
$$

where $\mathbf{y}_{k}^{[n]}=\left[\begin{array}{llll}y_{1, k}^{[n]} & y_{2, k}^{[n]} & \cdots & y_{T_{k}, k}^{[n]}\end{array}\right]^{\top}$ is a $T_{k} \times 1$ vector with $T_{k}$ elements representing all the measurements collected by user $k$ from anchor $n, \mathbf{s}_{k}^{[n]}=\left[\begin{array}{llll}s_{1, k}^{[n]} & s_{2, k}^{[n]} & \cdots & s_{T_{k}, k}^{[n]}\end{array}\right]^{\top}$ is a $T_{k} \times 1$ vector whose entries are defined by $s_{t, k}^{[n]}=\left|\mathbf{P} \mathbf{x}_{t, k}-\mathbf{l}^{[n]}\right|$, the matrix $\boldsymbol{\Psi}_{T_{k}, k}$ is a $T_{k} \times J$ matrix whose $t$ th row is given by $\boldsymbol{\psi}^{\top}\left(\mathbf{x}_{t, k}\right)$, and $\boldsymbol{\nu}_{k}^{[n]}=\left[\begin{array}{llll}\nu_{1, k}^{[n]} & \nu_{2, k}^{[n]} & \cdots & \nu_{T_{k}, k}^{[n]}\end{array}\right]^{\top}$ is a $T_{k} \times 1$ vector containing the measurement noise values. We note that the elements of the matrix $\boldsymbol{\Psi}_{T_{k}, k}$ are functions of the locations of the $k$ th user in the time interval 1 to $T_{k}, \mathbf{x}_{1: T_{k}, k}$. For the sake of notation simplicity we omit such dependence.

Next we suppose that the central unit keeps receiving the measurements $y_{t, k}^{[n]}, \forall n$. Based on these measurements, this unit has to estimate the state vector $\mathbf{x}_{t, k}$ and to update the estimate of the coefficients $\mathbf{c}^{[n]}, \forall n$. To that end, in [22] a "loose coupling" approach was proposed in which two separate methods for estimating the two sets of unknowns "communicate" with each other throughout the estimation. Here we propose a different and improved version of this approach.

\section{A NOVEL LOOSE COUPLING APPROACH}

We want to track the unknown process $\mathbf{x}_{t, k}$ and we note that this would require knowledge of $f^{[n]}\left(\mathbf{x}_{t, k}\right), \forall n$ (see (2)). According to our model, to know $f^{[n]}\left(\mathbf{x}_{t, k}\right)$ means basically to know the statistics of $\mathbf{c}^{[n]}$, i.e., $\boldsymbol{\mu}_{c}^{[n]}$ and $\boldsymbol{\Sigma}_{c}^{[n]}$. From (6), it is clear that the estimation of these statistics would be easy if we knew $\mathbf{x}_{t, k}$. We observe that we could design an algorithm that would estimate all of these unknowns jointly or we could use the principle divide and conquer and have two processing blocks, where each block is assigned simpler tasks. More specifically, one block would estimate $\boldsymbol{\mu}_{c}^{[n]}$ and $\boldsymbol{\Sigma}_{c}^{[n]}$, and the other would only track $\mathbf{x}_{t, k}$. We present an approach based on assigning the tasks to two separate processors, and we call this approach a loose coupling approach.

The tracker is based on a particle filtering (PF) algorithm [23], [24]. Let us assume that before we observe $\mathbf{y}_{t, k}$, we have an approximation of the marginal posterior $p\left(\mathbf{x}_{0: t-1, k} \mid \mathbf{y}_{1: t-1, k}\right)$ of 
the state sequence $\mathbf{x}_{0: t-1, k}$. The approximation is given by the probability random measure

$p^{M}\left(\mathbf{x}_{0: t-1, k} \mid \mathbf{y}_{1: t-1, k}, \widehat{\mathbf{c}}_{1: t-1, k}\right)=\sum_{m=1}^{M} w_{t-1, k}^{(m)} \delta\left(\mathbf{x}_{0: t-1, k}-\mathbf{x}_{0: t-1, k}^{(m)}\right)$,

where $\mathbf{x}_{0: t-1, k}^{(m)}$ is the $m$ th stream of particles, $w_{t-1, k}^{(m)}$ is the weight associated to the stream $\mathbf{x}_{0: t-1, k}^{(m)}, \delta(\cdot)$ is the Dirac delta function, $M$ is the total number of streams, and $\mathbf{y}_{1: t-1, k}$ and $\widehat{\mathbf{c}}_{1: t-1, k}$ represent all the observations $\mathbf{y}_{1: t-1, k}^{[n]}$, and estimates $\widehat{\mathbf{c}}_{1: t-1, k}^{[n]}, n=1,2, \ldots, N_{a}$. The particle filter is based on the model given by (1) and (6), where $\mathbf{c}^{[n]}$ is assumed known because it is obtained from the other processing block.

The filter propagates the particles $\mathbf{x}_{t-1, k}^{(m)}$ by

$$
\mathbf{x}_{t, k}^{(m)} \sim p\left(\mathbf{x}_{t, k} \mid \mathbf{x}_{t-1, k}^{(m)}\right),
$$

which is a Student's t-density (because of the unknown $\rho_{k}^{2}$ ), and then computes their weights according to

$$
w_{t, k}^{(m)} \propto \prod_{n=1}^{N_{a}} p\left(y_{t, k}^{[n]} \mid y_{1: t-1, k}^{[n]}, \mathbf{x}_{0: t, k}^{(m)}, \widehat{\mathbf{c}}_{1: t-1, k}^{[n]}, \mathcal{D}_{k-1}^{[n]}\right)
$$

where $p\left(y_{t, k}^{[n]} \mid y_{1: t-1, k}^{[n]}, \mathbf{x}_{0: t, k}^{(m)}, \widehat{\mathbf{c}}_{1: t-1, k}^{[n]}, \mathcal{D}_{k-1}^{[n]}\right)$ are t-densities of the form [25, p. 70]

$$
\begin{array}{r}
p\left(y_{t, k}^{[n]} \mid y_{1: t-1, k}^{[n]}, \mathbf{x}_{0: t, k}^{(m)}, \widehat{\mathbf{c}}_{1: t-1, k}^{[n]}, \mathcal{D}_{k-1}^{[n]}\right) \\
=\mathcal{T}\left(\bar{y}_{t, k}^{[n](m)}, \Upsilon_{t, k}^{[n](m)}, \kappa_{t-1, k}\right),
\end{array}
$$

with

$$
\begin{aligned}
\bar{y}_{t, k}^{[n](m)} & =\left|\mathbf{P} \mathbf{x}_{t, k}^{(m)}-\mathbf{l}^{[n]}\right|+\boldsymbol{\psi}^{\top}\left(\mathbf{x}_{t, k}^{(m)}\right) \widehat{\mathbf{c}}_{t-1, k}^{[n]}, \\
\Upsilon_{t, k}^{[n](m)} & =\frac{v_{t-1, k}^{[n](m)}}{\kappa_{t-1, k}}
\end{aligned}
$$

and

$$
\begin{aligned}
\kappa_{t-1, k} & =\kappa_{k-1}+\frac{1}{2}(t-1), \quad v_{t-1, k}^{[n](m)}=v_{k-1}^{[n]}+\sum_{\tau=1}^{t-1} r_{\tau, k}^{[n](m)}, \\
r_{\tau, k}^{[n](m)} & =\frac{1}{2}\left(y_{\tau, k}^{[n]}-\bar{y}_{\tau, k}^{[n](m)}\right)^{2} \\
\kappa_{k-1} & =\frac{1}{2} \sum_{i=1}^{k-1} T_{i}, \quad v_{k-1}^{[n]}=\sum_{i=1}^{k-1} \sum_{\tau=1}^{T_{i}} \sum_{m=1}^{M} w_{\tau, i}^{(m)} r_{\tau, i}^{[n](m)}
\end{aligned}
$$

The values $\mathcal{D}_{k-1}^{[n]}=\left\{\kappa_{k-1}, v_{k-1}^{[n]}\right\}$ account for the statistics of past users.

From the obtained weights and particles, the tracker computes the estimate of $\mathbf{x}_{t, k}$ according to

$$
\widehat{\mathbf{x}}_{t, k}=\sum_{m=1}^{M} w_{t, k}^{(m)} \mathbf{x}_{t, k}^{(m)} .
$$

This estimate, $\widehat{\mathbf{x}}_{t, k}$, is now passed on to the estimator of $\mathbf{c}^{[n]}$. The estimator works with "observations" given by

$$
\widehat{z}_{t, k}^{[n]}=y_{t, k}^{[n]}-\left|\mathbf{P} \widehat{\mathbf{x}}_{t, k}-\mathbf{l}^{[n]}\right| .
$$

We can express $\widehat{z}_{t, k}^{[n]}$ by

$$
\widehat{z}_{t, k}^{[n]}=\boldsymbol{\psi}^{\top}\left(\widehat{\mathbf{x}}_{t, k}\right) \mathbf{c}_{t-1, k}^{[n]}+\nu_{t, k}^{[n]}, \quad n=1,2, \cdots, N_{a} .
$$

Because the unknown coefficients are linear parameters given the trajectory of the user, we readily deduce that we can easily update them with recursive least-squares equations by [26]

$$
\widehat{\mathbf{c}}_{t, k}^{[n]}=\widehat{\mathbf{c}}_{t-1, k}^{[n]}+\mathbf{k}_{t}^{[n]}\left(\widehat{z}_{t, k}^{[n]}-\boldsymbol{\psi}^{\top}\left(\widehat{\mathbf{x}}_{t, k}\right) \widehat{\mathbf{c}}_{t-1, k}^{[n]}\right),
$$

where $\mathbf{k}_{t}^{[n]}$ is a $J \times 1$ vector defined by

$$
\mathbf{k}_{t}^{[n]}=\frac{\boldsymbol{\Sigma}_{c, t-1}^{[n]} \boldsymbol{\psi}\left(\widehat{\mathbf{x}}_{t, k}\right)}{1+\boldsymbol{\psi}^{\top}\left(\widehat{\mathbf{x}}_{t, k}\right) \boldsymbol{\Sigma}_{c, t-1}^{[n]} \boldsymbol{\psi}\left(\widehat{\mathbf{x}}_{t, k}\right)},
$$

where

$$
\boldsymbol{\Sigma}_{c, t}^{[n]}=\left(\mathbf{I}-\mathbf{k}_{t}^{[n]} \boldsymbol{\psi}^{\top}\left(\widehat{\mathbf{x}}_{t, k}\right)\right) \boldsymbol{\Sigma}_{c, t-1}^{[n]} .
$$

The new estimates $\widehat{\mathbf{c}}_{t, k}^{[n]}$ are communicated to the tracker, which proceeds with estimating $\mathbf{x}_{t+1, k}$.

\section{III-A. Summary of statistics of past users}

When the $k$ th user leaves the area, the central unit keeps a summary $\mathcal{D}_{k}^{[n]}=\left\{\kappa_{k}, v_{k}^{[n]}\right\}$ of the statistics of the previous users for $n=1,2, \ldots N_{a}$. One set of statistics critical for computing the weight coefficients and deduced from (15) is given by

$$
\begin{aligned}
\kappa_{k} & =\kappa_{k-1}+\frac{1}{2} T_{k}, \\
v_{k}^{[n]} & =v_{k-1}^{[n]}+\sum_{\tau=1}^{T_{k}} \sum_{m=1}^{M} w_{\tau, k}^{(m)} r_{\tau, k}^{[n](m)},
\end{aligned}
$$

$\forall n$, and where $\kappa_{0}=0$ and $v_{0}^{[n]}=0$. In total, the system keeps $N_{a}+1$ statistics for tracking the state.

For the field estimates, the system needs to keep $\widehat{\mathbf{c}}_{T_{k}, k}^{[n]}$ and $\boldsymbol{\Sigma}_{c, T_{k}}^{[n]}$ $\forall n$. Therefore, the system maintains a total of $N_{a}$ vectors of size $J \times 1$ and $N_{a}$ covariance matrices of size $J \times J$.

As already pointed out, the size of the above statistics neither depends on the number of users that have provided measurements nor on the total number of measurements.

\section{NUMERICAL RESULTS}

Now we present some numerical results in which the observation values are based on real measurements. In particular, the scenario where the measurement campaign was carried out is shown in Fig. 1 , which represents a typical office indoor environment with walls made of concrete. We have considered 4 anchors, denoted in the figure as $\mathrm{tx}_{i}, i=\{1,2,3,4\}$. The figure shows also a set of 20 locations where 1500 range measurements were taken for each anchor using a commercial ultrawide-band (UWB) radio operating in the $3.2-7.4 \mathrm{GHz}$ band. The detailed setup of the experiment is described in [16]. For each anchor and each of the 20 test locations, the mean and the standard deviation of the range measurements were computed. Then the bias for each pair anchor-test location was obtained as the difference between the mean range observation and the true distance. The result of interpolating this set of bias values at a given location was considered as the true bias field for that location for simulation purposes. As can be noticed, most test locations are in NLOS with respect to the anchors, thus making the localization and fields estimation processes quite challenging.

In the simulations, we allowed users to move randomly in a square area of side $L=9$ meters in Fig. 1. Regarding the 2D orthogonal basis function $\psi(\mathbf{x})$ used by the learning process, we considered the 2D Fourier series expansion of the periodical 




Fig. 1. Floor plan of the office environment considered in the case study. The locations of the anchors and the test locations are shown.

repetition of $f^{[n]}(\mathbf{x})$ with a period $L$ in each dimension and truncated to $J=81$ terms. A total of 50 measurements were taken for each user during his/her movement at intervals of $1 \mathrm{~s}$. The covariance of the random perturbation in (1) was $\mathbf{R}_{k}=\rho_{k}^{2} \mathbf{I}$, where $\rho_{k}=0.1$ and $\mathbf{I}$ is the identity matrix. The measurement noise variance was $\sigma_{\nu}^{[n]}=0.1 \mathrm{~m}$. For each user, we ran the proposed algorithm with 500 particles and the method in [22] with an extended Kalman filter (EKF). For comparison, a simple technique assuming always LOS (and thus, zero bias) and a method with perfect knowledge of bias at every instant, both based on EKF, were also considered. This last method is unrealistic and is included as a benchmark. The process was repeated for 500 Monte Carlo iterations. For each iteration, the crowd based methods started without any knowledge about the true bias field, taking for the first user as initial value $\widehat{\mathbf{c}}_{0,0}^{[n]}$ a vector of zeros and $\boldsymbol{\Sigma}_{c, 0}^{[n]}=\mathbf{I}$.

It is worth noting that in [22] an initial training phase was considered in which several collaborating users navigated the area along prescribed trajectories. By contrast, in this paper we focus on the challenging situation where no training phase is considered. The results in terms of (empirical) cumulative distribution function (CDF) are shown in Figs. 2-3. These curves encompass all the values of localization error (i.e., distance between true and estimated location) for each of the 50 measurements per user and each of the 500 iterations.

Specifically, Fig. 2 shows the results for the crowd-based method with EKF in [22]. It can be observed that as the number of users grows, the crowd mapping approach outperforms the simple method assuming LOS.

The results for the crowd-based method with PF proposed in this paper are reported in Fig. 3. These results are remarkably better than those in Fig. 2, even from the beginning of the crowd sensing process (a few users). For example, if one sets a target performance at $0.5 \mathrm{~m}$, with the new loose approach, after only 6 users having navigated in the area, more than $41 \%$ of locations meet the target performance (covered) with respect to $26 \%$ assuming

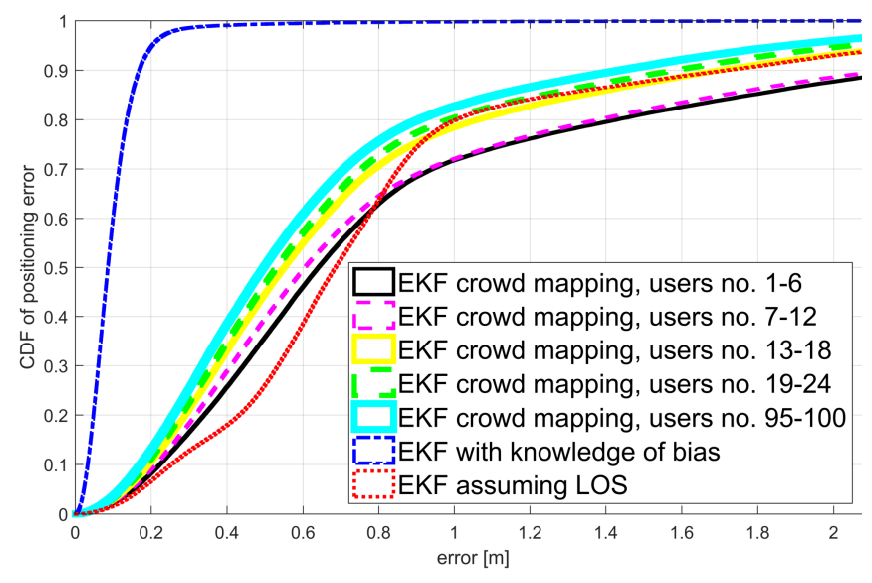

Fig. 2. CDF of positioning error for the crowd-based method in [22] with EKF in the absence of any calibration.

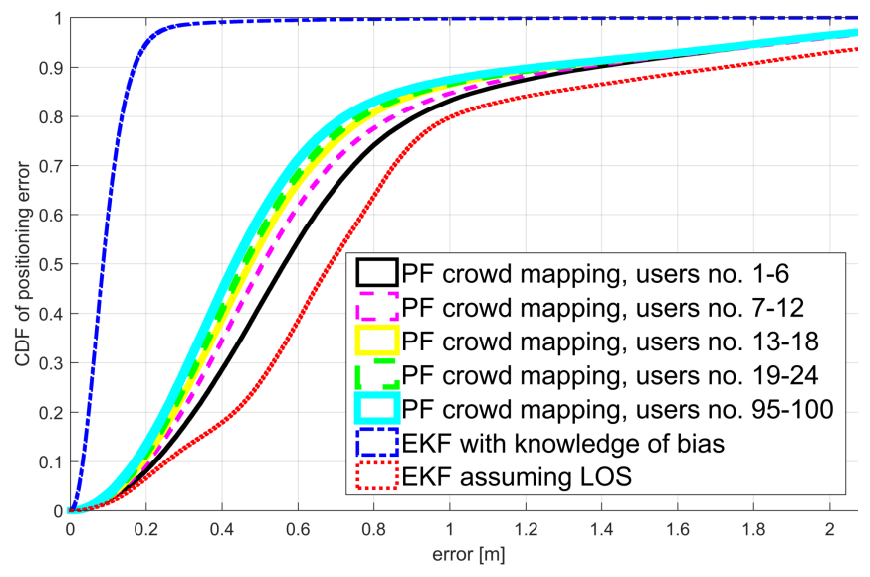

Fig. 3. CDF of positioning error for the proposed crowd-based method with $\mathrm{PF}$ in the absence of any calibration.

always LOS and $36 \%$ with the method in [22]. As the number of users grows, a progressive improvement of performance of the crowd-based method can be observed. The results show that about $60 \%$ of the area has the same target performance after 100 users.

\section{CONCLUSIONS}

Simulation results, based on real measurements, confirm that the proposed method is capable of enhancing the knowledge about the fields of NLOS-induced bias even in the challenging case of absence of any calibration. Additionally, the complexity and memory requirements do not grow with the number of users and measurements. The results in terms of CDF show that the proposed method improves the localization performance as the number of users increases, outperforming a previously proposed crowd mapping method without any calibration. Despite this remarkable result, the gap between the ideal case of perfect knowledge of the bias and our method suggests that there is room for further improvement in future work, for example, by using available information on the floor plan. 


\section{REFERENCES}

[1] P. Bellavista, A. Küpper, and S. Helal, "Location-based services: Back to the future," IEEE Pervasive Computing, vol. 7, no. 2, pp. 85-89, 2008.

[2] H. Liu, H. Darabi, P. Banerjee, and J. Liu, "Survey of wireless indoor positioning techniques and systems," IEEE Transactions on Systems, Man, and Cybernetics, Part C (Applications and Reviews), vol. 37, no. 6, pp. 1067-1080, 2007.

[3] D. Dardari, P. Closas, and P. Djuric, "Indoor tracking: Theory, methods, and technologies," IEEE Trans. Veh. Technol., vol. 64, no. 4, pp. 1263-1278, Apr. 2015.

[4] B. Alavi and K. Pahlavan, "Modeling of the TOAbased distance measurement error using UWB indoor radio measurements," IEEE Communications Letters, vol. 10, no. 4, pp. 275-277, Apr. 2006.

[5] M. Heidari and K. Pahlavan, "A new statistical model for the behavior of ranging errors in TOA-based indoor localization," in 2007 IEEE Wireless Communications and Networking Conference, March 2007, pp. 2564-2569.

[6] E. Arias-de Reyna, "A cooperative localization algorithm for UWB indoor sensor networks," Wireless Personal Communications, vol. 72, no. 1, pp. 85-99, Sep 2013.

[7] E. Arias-de Reyna and U. Mengali, "A maximum likelihood UWB localization algorithm exploiting knowledge of the service area layout," Wireless Personal Communications, vol. 69, no. 4, pp. 1413-1426, Apr 2013.

[8] C. Morelli, M. Nicoli, V. Rampa, and U. Spagnolini, "Hidden markov models for radio localization in mixed LOS/NLOS conditions," IEEE Trans. on Signal Processing, vol. 55, no. 4, pp. 1525-1542, April 2007.

[9] M. A. Caceres and F. Sottile and M. A. Spirito, "Adaptive location tracking by Kalman filter in wireless sensor networks," in 2009 IEEE International Conference on Wireless and Mobile Computing, Networking and Communications. IEEE, 2009, pp. 123-128.

[10] K. Lui, H. So, and W.-K. Ma, "Maximum a posteriori approach to time-of-arrival-based localization in non-line-ofsight environment," IEEE Trans. on Vehicular Technology, vol. 59, no. 3, pp. 1517-1523, March 2010.

[11] Y. Shen, H. Wymeersch, and M. Win, "Fundamental limits of wideband localization - part II: Cooperative networks," IEEE Trans. on Information Theory, vol. 56, no. 10, pp. 4981 5000, Oct. 2010.

[12] L. Chen, S. Ali-Löytty, R. Piché, and L. Wu, "Mobile tracking in mixed line-of-sight/non-line-of-sight conditions: Algorithm and theoretical lower bound," Wireless Personal Communications, vol. 65, no. 4, pp. 753-771, 2012.

[13] Y. Liu, M. Dashti, M. Abd Rahman, and J. Zhang, "Indoor localization using smartphone inertial sensors," in 2014 11th Workshop on Positioning, Navigation and Communication (WPNC), March 2014, pp. 1-6.

[14] J. M. Castro-Arvizu, J. Vilà-Valls, A. Moragrega, P. Closas, and J. A. Fernández-Rubio, "Received signal strength-based indoor localization using a robust interacting multiple modelextended Kalman filter algorithm," International Journal of Distributed Sensor Networks, vol. 13, no. 8, 2017.

[15] N. E. Gemayel, J. Schloemann, R. M. Buehrer, and F. K. Jondral, "Improved indoor positioning using the Baum-Welch algorithm," in IEEE Globecom 2015, Dec. 2015.

[16] D. Dardari, A. Conti, J. Lien, and M. Z. Win, "The effect of cooperation on localization systems using UWB experimental data," EURASIP Journal on Advances in Signal Processing, Special Issue on Cooperative Localization in Wireless Ad Hoc and Sensor Networks, vol. 2008, 2008, article ID 513873, 12 pages.

[17] S. Marano, W. M. Gifford, H. Wymeersch, and M. Z. Win, "NLOS identification and mitigation for localization based on UWB experimental data," IEEE Journal on Selected Areas in Communications, vol. 28, no. 7, pp. 1026-1035, September 2010.

[18] H. Ma, D. Zhao, and P. Yuan, "Opportunities in mobile crowd sensing," IEEE Communications Magazine, vol. 52, no. 8, pp. 29-35, 2014.

[19] L. Pei, M. Zhang, D. Zou, R. Chen, and Y. Chen, "A survey of crowd sensing opportunistic signals for indoor localization," Mobile Information Systems, vol. 2016, 2016.

[20] E. S. Lohan, J. Torres-Sospedra, H. Leppäkoski, P. Richter, Z. Peng, and J. Huerta, "Wi-Fi Crowdsourced Fingerprinting Dataset for Indoor Positioning," Data, vol. 2, no. 4, p. 32, 2017.

[21] S.-J. Kim, E. Dall'Anese, and G. B. Giannakis, "Cooperative spectrum sensing for cognitive radios using Kriged Kalman filtering," IEEE Journal of Selected Topics in Signal Processing, vol. 5, no. 1, pp. 24-36, 2011.

[22] E. Arias-de-Reyna, D. Dardari, P. Closas, and P. M. Djurić, "Enhanced indoor localization through crowd sensing," in 2017 IEEE International Conference on Acoustics, Speech and Signal Processing (ICASSP), March 2017, pp. 24872491.

[23] P. M. Djurić, J. H. Kotecha, J. Zhang, Y. Huang, T. Ghirmai, M. F. Bugallo, and J. Míguez, "Particle filtering," IEEE Signal Processing Magazine, vol. 20, no. 5, pp. 19-38, 2003.

[24] A. Doucet and A. M. Johansen, "A tutorial on particle filtering and smoothing: Fifteen years later," in Handbook of Nonlinear Filtering, D. Crisan and Rozovskii, Eds., 2011, pp. 656-704.

[25] A. Zellner, An Introduction to Bayesian Inference in Econometrics. John Wiley \& Sons, 1971.

[26] S. M. Kay, Fundamentals of Statistical Signal Processing: Estimation Theory. Upper Saddle River, NJ, USA: PrenticeHall, Inc., 1993. 